\title{
Novel human lipoxygenase inhibitors discovered using virtual screening with homology models
}

\author{
Victor Kenyon ${ }^{\#}$, Ilya Chorny ${ }^{\S}$, Wendy J. Carvajal ${ }^{\#}$, Theodore R. Holman ${ }^{\# 1}$, \\ and Matthew P. Jacobson ${ }^{\S 2}$
}

$\S$ Department of Pharmaceutical Chemistry, University of California, San Francisco, 94143-2240

\# Department of Chemistry and Biochemistry, University of California, Santa Cruz 95064

\section{Supporting Information}

\footnotetext{
${ }^{1}$ To whom the correspondence should be addressed: Telephone: 831-459-5884. Fax: 831-459-4823. Email: tholman@chemistry.ucsc.edu

2 To whom the correspondence should be addressed: Telephone: 415-514-9811. Fax: 415-514-4260. Email: matt@cgl.ucsf.edu
} 
Supplementary Table 1. Structures, ranks from Glide SP docking, and estimated $\mathrm{IC}_{50}$ values for the randomly selected compounds. Some compounds did not successfully dock into one or both of the models, generally due to a steric clash or other highly unfavorable interaction. "Agg” refers to compounds that displayed visible aggregation, despite the use of detergent in the assay.

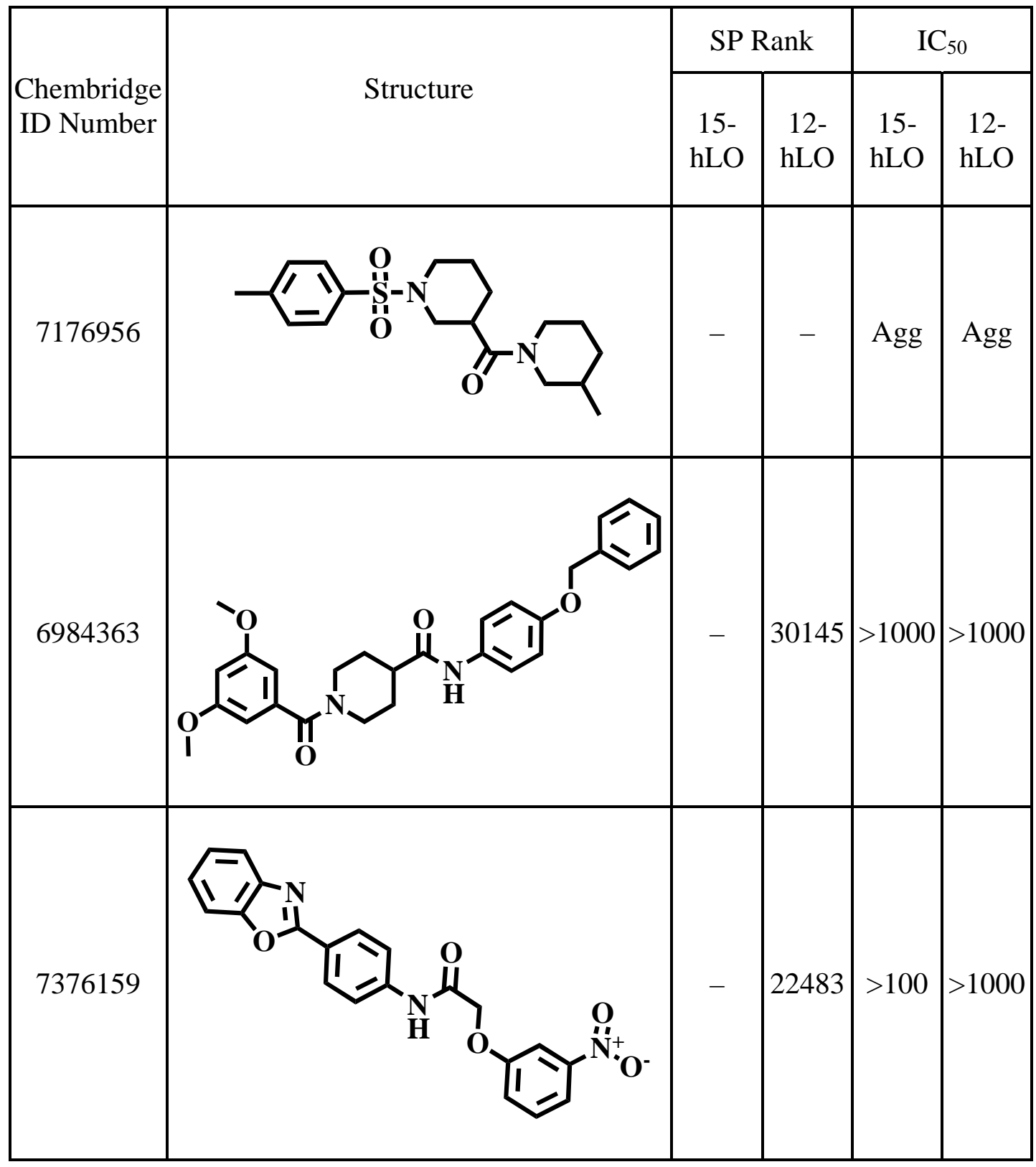




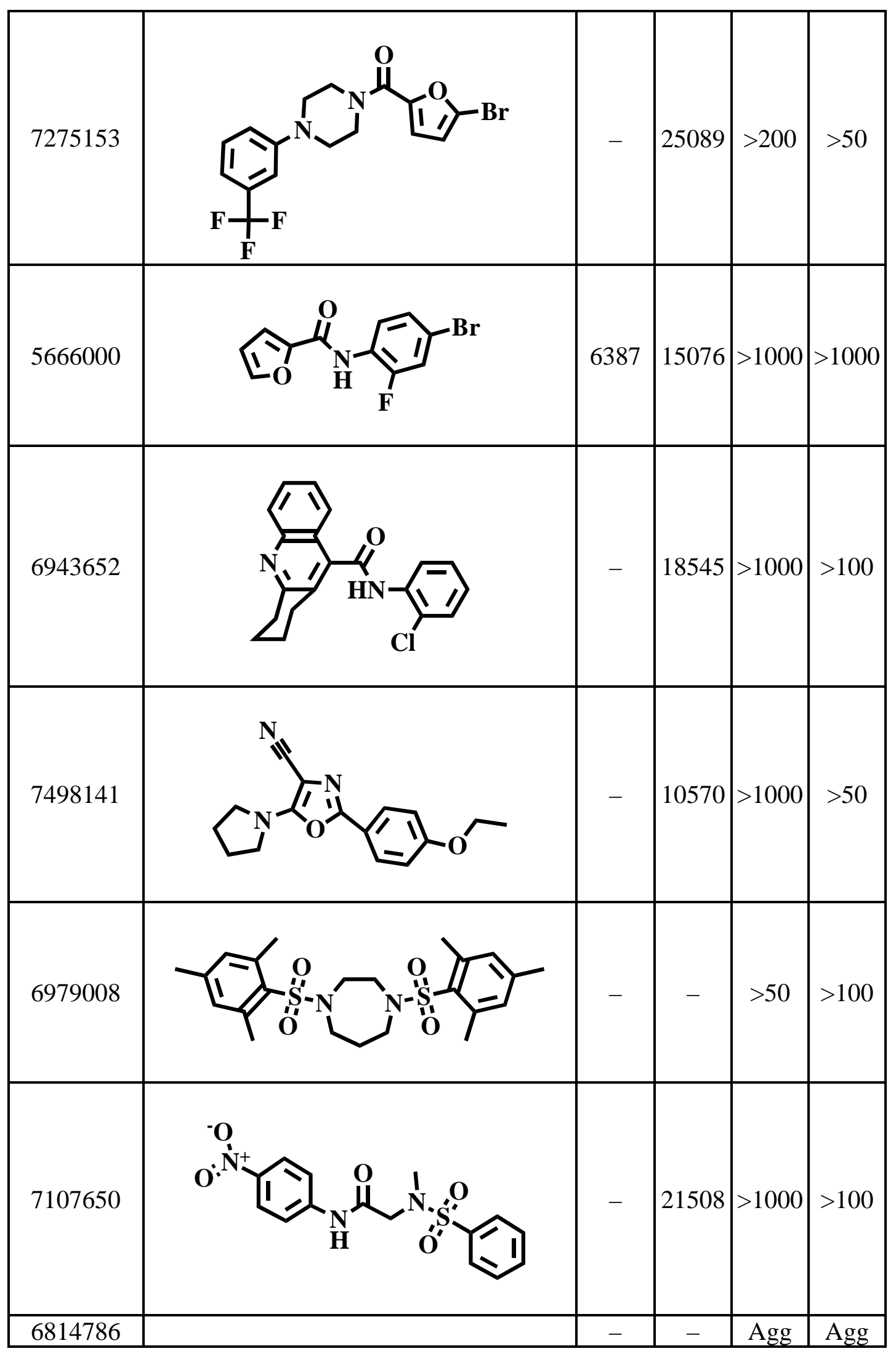




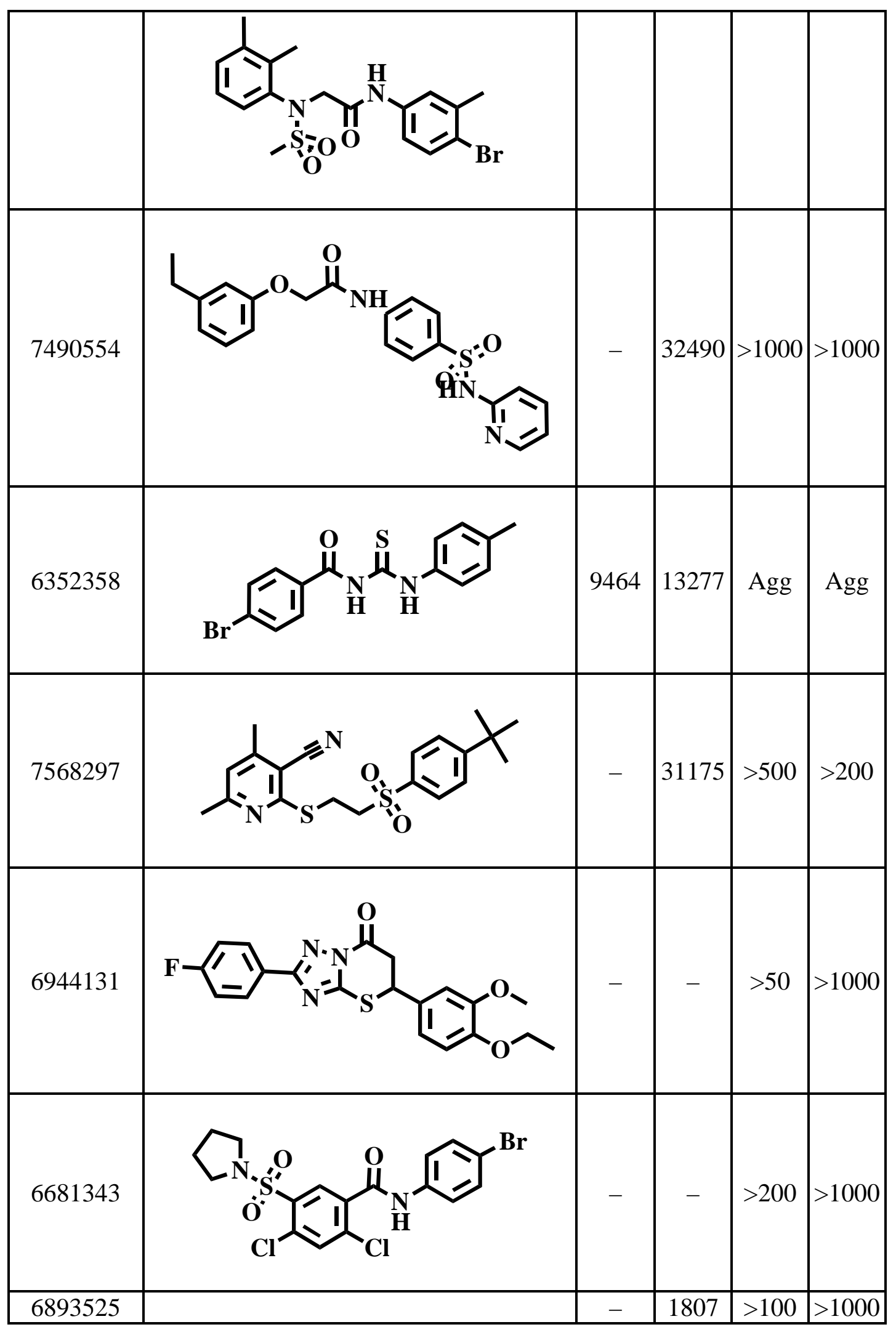




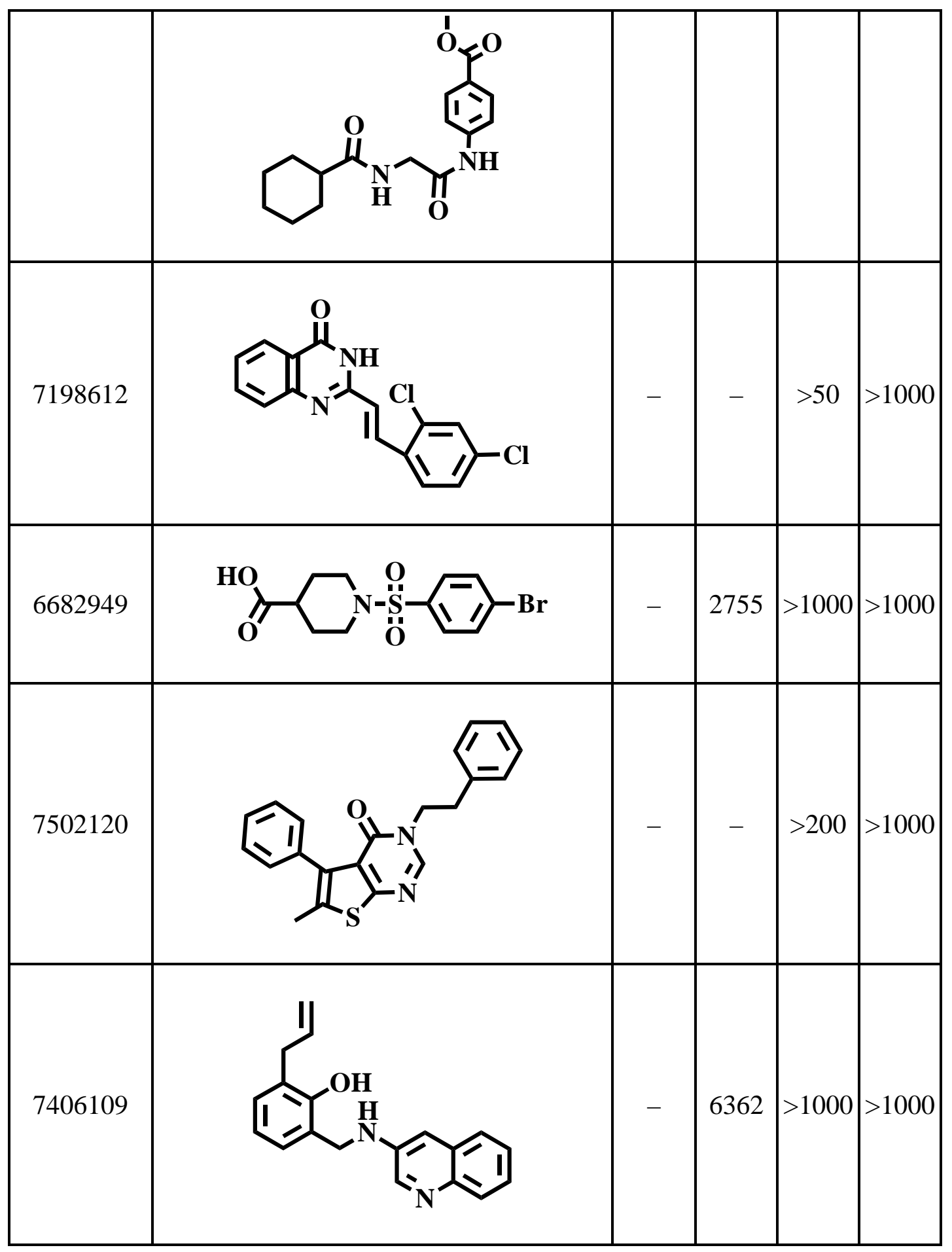




\section{Supplementary Figures 1-3:}

Inhibition Fits. IC 50 values were calculated by using a standard hyperbolic equation $\mathrm{y}=$ $(\mathrm{m} 0 * \mathrm{~m} 1) /(\mathrm{m} 0+\mathrm{m} 2)$, where $\mathrm{y}=$ percent inhibition, $\mathrm{m} 0=$ inhibitor concentration ([inhibitor]), $\mathrm{m} 1=$ percent inhibition at infinite inhibitor concentration and $\mathrm{m} 2=\mathrm{IC}_{50}$.

1) $\mathrm{IC}_{50}$ fits for the inhibitor h15-009 against15-hLO (a) and 12-hLO (b)

(a)

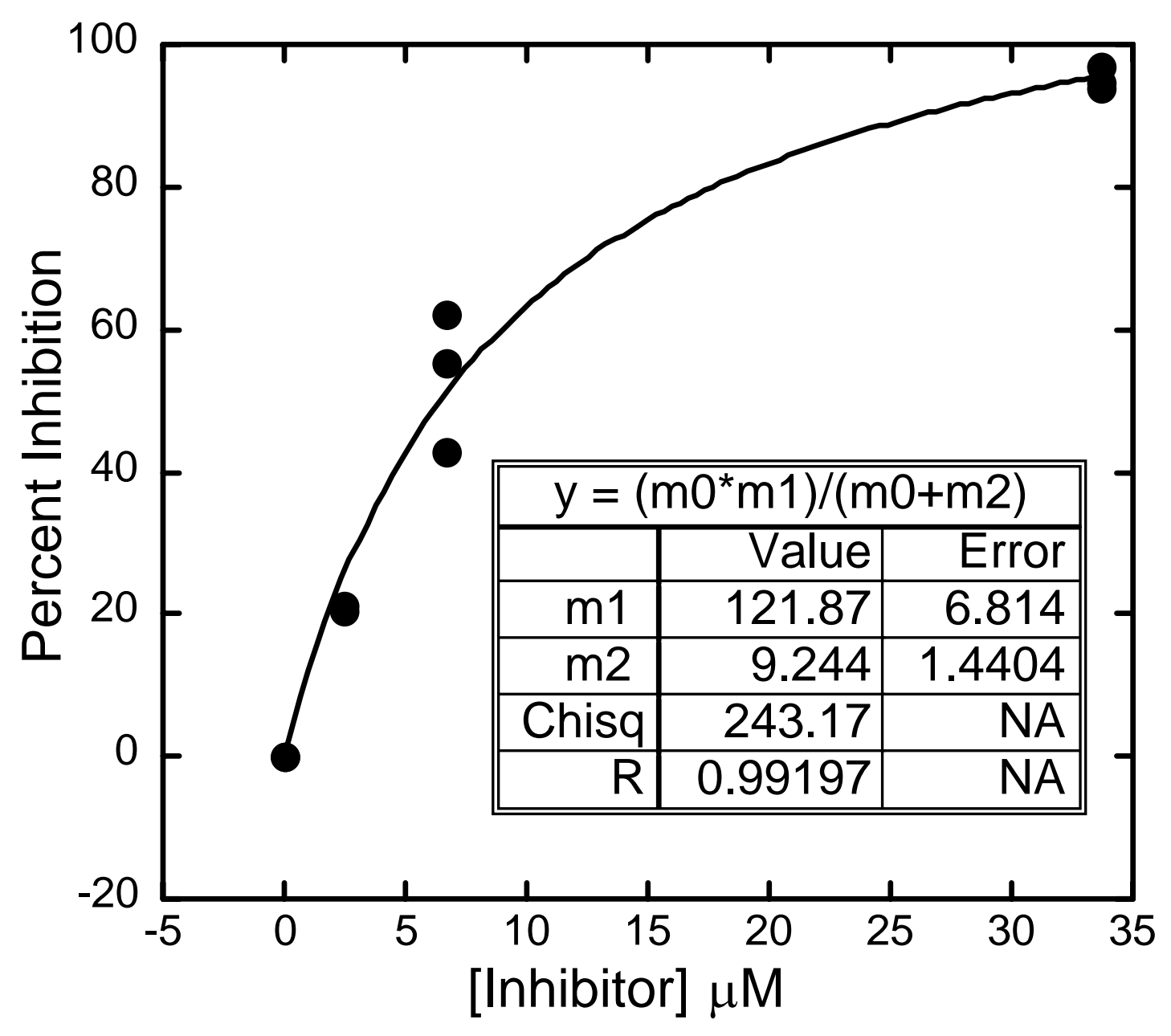


(b)

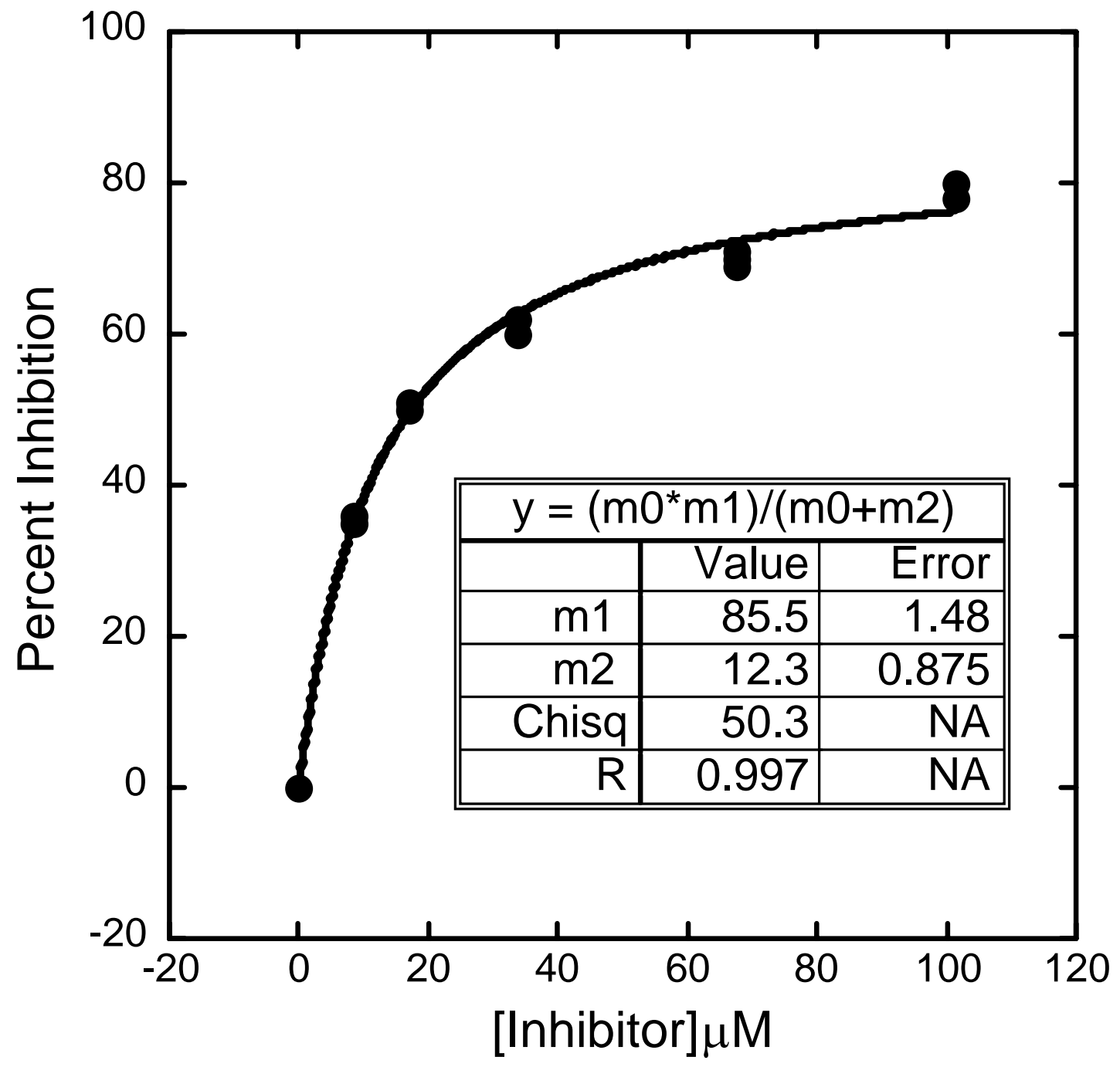


2) $\mathrm{IC}_{50}$ fits for the inhibitor h12-002 against15-hLO (a) and 12-hLO (b)

(a)

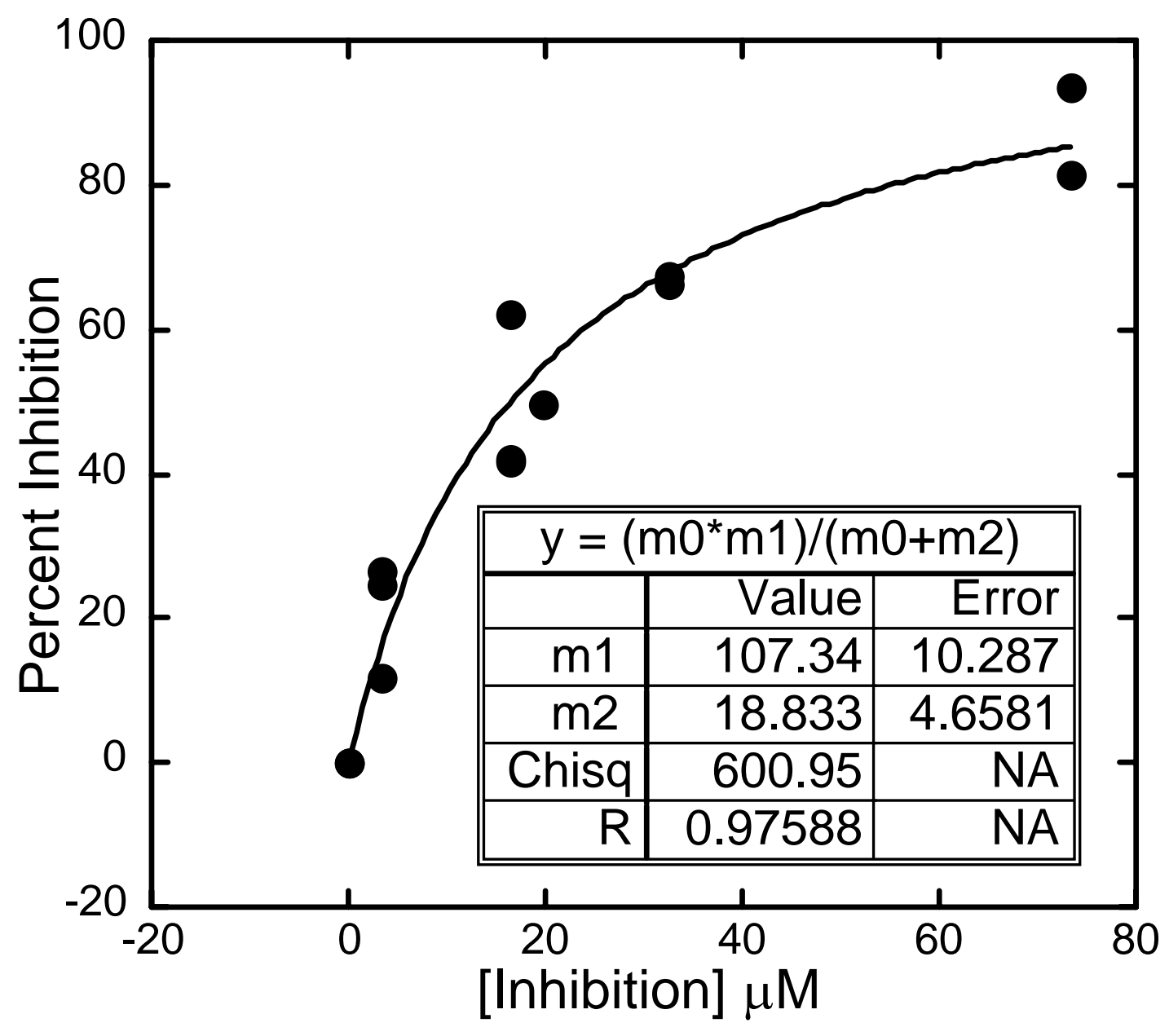


(b)

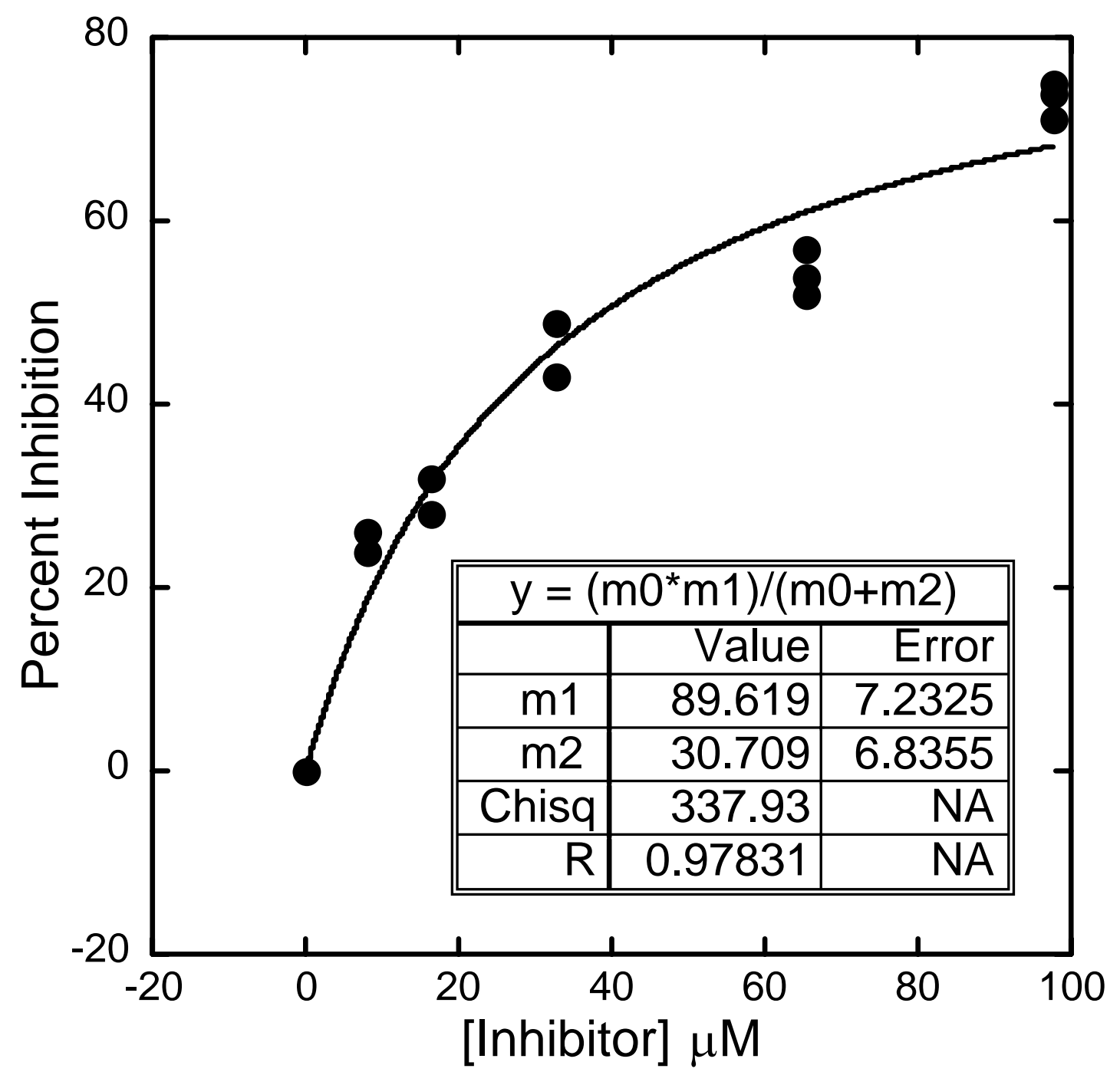


3) $\mathrm{IC}_{50}$ fit for the inhibitor h12-005 against15-hLO. No appreciable inhibition was observed against 12-hLO ( $\left.\mathrm{IC}_{50}>200 \mu \mathrm{M}\right)$

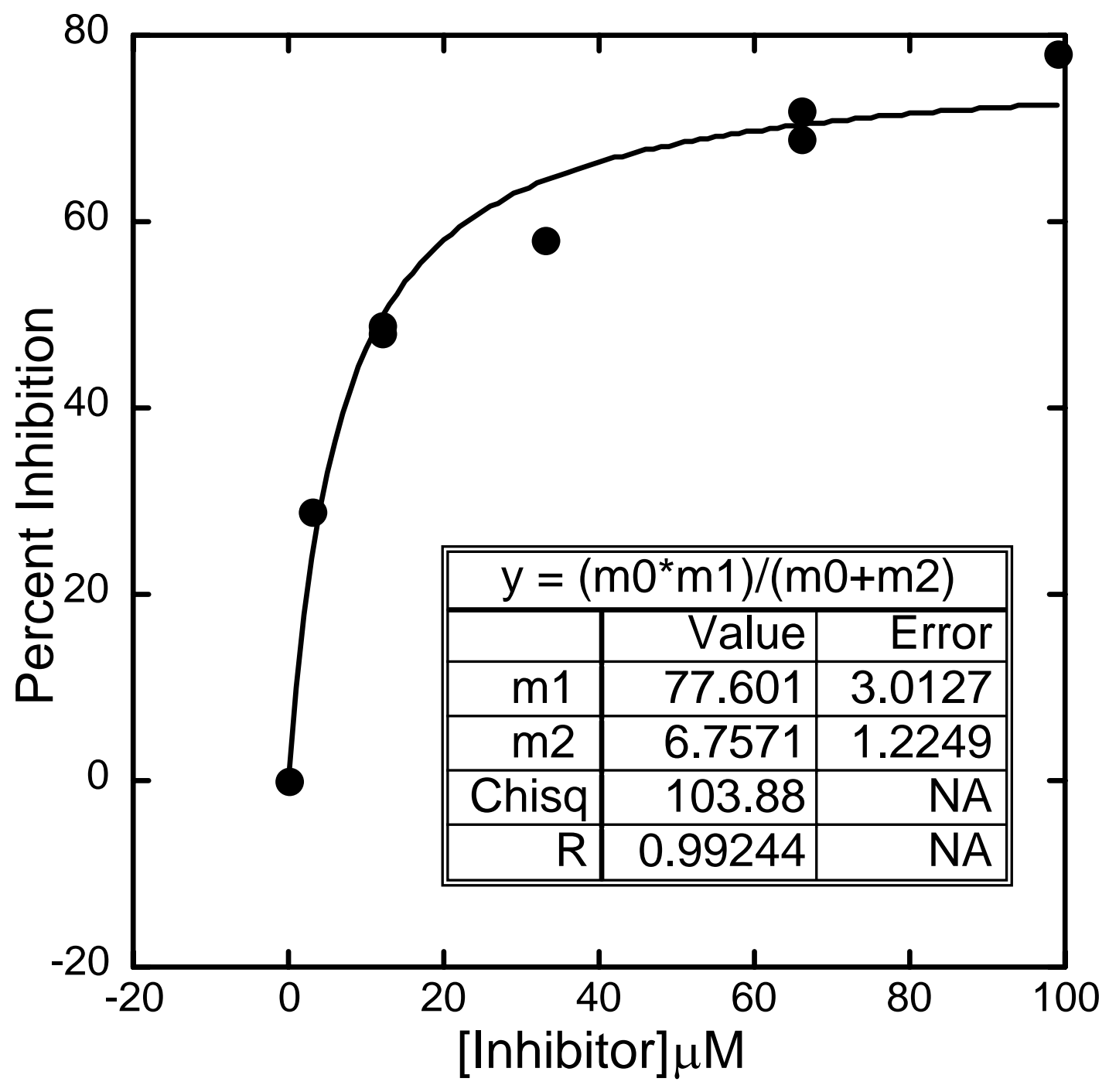

